\title{
Asymptotic Properties of Optimized Type CVaR Estimator for NA Random Variables
}

\author{
Shanchao Yang ${ }^{1}$, Yuting Wang ${ }^{1}$, Xin Yang ${ }^{2,}$, Xiutao Yang ${ }^{3}$ \\ ${ }^{1}$ School of Mathematics and Statistics, Guangxi Normal University, Guilin, China \\ ${ }^{2}$ School of Mathematical Sciences, Guilin University of Aerospace Technology, Guilin, China \\ ${ }^{3}$ Department of Basic Course Teaching, Haikou College of Economics, Haikou, China \\ Email address: \\ scyang@mailbox.gxnu.edu.cn (Shanchao Yang), 1209030930@qq.com (Yuting Wang), xinyang_emily@sina.cn (Xin Yang) \\ *Corresponding author
}

\section{To cite this article:}

Shanchao Yang, Yuting Wang, Xin Yang, Xiutao Yang. Asymptotic Properties of Optimized Type CVaR Estimator for NA Random Variables. American Journal of Theoretical and Applied Statistics. Vol. 8, No. 6, 2019, pp. 253-260. doi: 10.11648/j.ajtas.20190806.17

Received: October 8, 2019; Accepted: November 9, 2019; Published: November 25, 2019

\begin{abstract}
VaR and CVaR are important risk measures, which are widely used in finance, economy, insurance and other fields. However, VaR is not a coherent risk quantity, and it is not sufficient to measure tail risk. CVaR (also known as expected shortfall, ES) is a coherent risk measure, and it makes up for the defect that VaR is not enough to measure tail risk. Therefore, CVaR has been paid more and more attention in both application and theory fields. Rockafellar and Uryasev (2000) and Trindade et al (2007) proposed an optimized type CVaR estimator and studied some asymptotic properties of the estimator. Since then, some scholars have discussed the properties of the estimator in the cases of $\rho$-mixing, $\varphi$-mixing and $\alpha$-mixing. In this paper, we shall study the asymptotic properties of the optimized type CVaR estimator in the case where the samples are NA random variables. The consistency and the asymptotic normality of the optimized type CVaR estimator and their corresponding convergence rates are obtained. The convergence rates of estimation are $n^{-1 / 2}$ or near to $n^{-1 / 2}$. These results also establish the asymptotic relations of the optimized type CVaR estimator and the common CVaR estimator. And their deviation converges almost surely to 0 at the rate of $n^{-1 / 2}$.
\end{abstract}

Keywords: CVaR Estimator, Consistency, Asymptotic Normality, Convergence Rate, NA Sequence

\section{Introduction}

In the fields of finance, economy and insurance, it is very important to measure risk accurately. In financial filed, VaR is widely used to measure risk, which refers to the maximum possible loss under a certain confidence level for financial assets or portfolios in the holding period. Let $X$ be a loss variable of financial asset or portfolio, for a given small probability $\alpha \in(0,1)$, in the confidence level of $1-\alpha$, VaR is defined as

$$
\operatorname{VaR}_{\alpha}(X)=\inf \{u: F(u) \geq 1-\alpha\},
$$

where $F(u)$ is distribution function of loss variable.

However, Artzner et al. [2-3] found that the VaR doesn't satisfy subadditivity, namely, it hasn't convexity. So VaR is not a coherent risk measure, and it is insufficient to measure the tail risk. As an alternative measure of risk, CVaR (also known as expected shortfall, ES) is proposed, also known as the average excess loss, which denotes the expected loss which greater than the given VaR value for a portfolio, and its formula as

$$
\operatorname{CVaR}_{\alpha}(X)=E\left(X \mid X \geq \operatorname{VaR}_{\alpha}(X)\right) .
$$

Rockafellar and Uryasev [15] proved that the definition of CVaR is equivalent to the solution of an optimization problem, namely

$$
\operatorname{CVaR}_{\alpha}(X)=\inf _{x \in R}\left\{x+\alpha^{-1} E[X-x]^{+}\right\},
$$

where $[a]^{+}=\max \{0, a\}$. Pflug [14] proved the CVaR satisfies subadditivity, also is a coherent risk measurement. More researches are available in Rockafellar and Uryasev [16], Föllmer and Schied [6].

CVaR has better properties than VaR. Therefore, it is suitable as the optimization tool of investment portfolio, 
which attracts scholar's widely attention. For example, Andrew et al. [1], Mansini et al. [12], Noyan and Rodulf [13], Sun et al. [22]. Noyan and Rodulf [13] extended to multivariate $\mathrm{CVaR}$, expand its application range. Also in other fields it is widely used, such as risk issues of books and newspapers selling (Gotoh and Takano, [7]), optimal placement of the gas detector in the petrochemical plant (Legg et al., [8]), wind power generation system (Chen et al., [4]), and so on.

Scaillet [18], Luo and Yang [10], Luo and Ou [11] and references therein studied the CVaR kernel estimator. Escanciano and Mayoral [5], Wang and Zhao [24] studied the CVaR semi-parametric estimation. On the basis of the equivalence definition of formula (3), Trindade et al. [23] proposed a kind of optimization CVaR estimation, and studied the consistency and the asymptotic normality of the optimization CVaR estimation. For the optimization CVaR estimation, Luo and Yang [10] studied the strong consistency and asymptotic normality under the $\rho$-mixing samples, and its convergence rate. Xing et al. [25] studied the strong consistency under the $\phi$-mixing sample, and gave the rate of convergence. However, negatively associated (NA) sequence is also common in dependence. It was proposed by Joag-Dev in 1983, and then attracts many scholars, such as Su Chun [20], Roussas [17], Yang [27], Zhou et al. [28] and so on. However, as we know, the asymptotic normality on the optimal CVaR estimation under NA sequence hasn't been studied, so this article will focus on the consistence and asymptotic normality of optimization CVaR estimation under NA sequence.

In the end of this section, we give some relevant definitions as follows.

The random variables $X_{1}, X_{2}, \cdots, X_{n}, n \geq 2$ are said to be negatively associated (NA), if, for two arbitrary disjoint subsets $A_{1}$ and $A_{2}$ of $\{1,2, \cdots, n\}$,

$$
\operatorname{Cov}\left(f_{1}\left(x_{i}, i \in A_{1}\right), f_{2}\left(x_{j}, j \in A_{2}\right)\right) \leq 0,
$$

where $f_{1}$ and $f_{2}$ are non-increasing or nondecreasing functions. Random sequence $\left\{X_{n}, n \geq 1\right\}$ is said to be NA if
$X_{1}, X_{2}, \cdots, X_{n}$ are NA for any $n \geq 2$.

Let $h_{\alpha}(X, x)=x+\alpha^{-1}[X-x]^{+}, \theta^{*}=\operatorname{CVaR}_{\alpha}(X)$, from the (3) we have

$$
\theta^{*}=\inf _{x \in \mathbb{R}} E h_{\alpha}(X, x) .
$$

Assume $X_{1}, X_{2}, \cdots, X_{n}$ is a sample coming from the population $X$, Rockafellar and Uryasev [15] and Trindade et al [23] define the optimization estimation for $\theta^{*}$, namely

$$
\hat{\theta}_{n}=\inf _{x \in \mathbb{R}} n^{-1} \sum_{i=1}^{n} h_{\alpha}\left(X_{i}, x\right) .
$$

\section{Main Result}

To describe our main results, we firs introduce some denotations. For any given $x \in \mathbb{R}$, let

$$
\begin{aligned}
& Z_{i}(x)=h_{\alpha}\left(X_{i}, x\right), g_{n}(x)=\frac{1}{n} \sum_{i=1}^{n} h_{\alpha}\left(X_{i}, x\right)=\frac{1}{n} \sum_{i=1}^{n} Z_{i}(x), \\
& S_{n}(x)=\sigma_{n}^{-1}(x)\left(g_{n}(x)-E g_{n}(x)\right), F_{n}^{x}(u)=P\left(S_{n}(x)<u\right),
\end{aligned}
$$

where $\sigma_{n}^{2}(x)=\operatorname{Var}\left(g_{n}(x)\right)$.

We need the following basic assumptions.

(A1) $\left\{X_{i}: i \geq 1\right\}$ is a sequence of identically distributed NA random variables with $E X_{1}=0$ and $E\left(\left|X_{1}\right|^{2+\delta}\right)<\infty$ for some $\delta>0$.

(A2)

$$
u(1)<\infty
$$

where

$u(n)=\sup _{j \geq 1} \sum_{i:|i-j| \geq n}\left|\operatorname{Cov}\left(X_{i}, X_{j}\right)\right|$.

(A3) $\sigma^{2}(x)>0$ for any $x \in \mathbb{R}$, where $\sigma^{2}(x)=$ $\lim _{n \rightarrow \infty} n \sigma_{n}^{2}(x)$.

(A4) There exist positive integers $p_{n}$ and $q_{n}$ such that

$$
p_{n}+q_{n} \leq n, q_{n} p_{n}^{-1} \leq C<\infty,
$$

and, as $n \rightarrow \infty$,

$$
\gamma_{1 n} \rightarrow 0, \gamma_{2 n} \rightarrow 0, u\left(q_{n}\right) \rightarrow 0 \text {. }
$$

where $\gamma_{1 n}=q_{n} p_{n}^{-1}, \gamma_{2 n}=p_{n} n^{-1}$.

Because $h_{\alpha}(X, x)$ is monotone increasing about $X$, $\left\{Z_{i}(x): i \geq 1\right\}$ is also a NA sequence. Under the assumptions (A1) and (A2), we have that

$$
\begin{aligned}
n \sigma_{n}^{2}(x) & =n^{-1} \sum_{i=1}^{n} \operatorname{Var}\left(Z_{i}(x)\right)+2 n^{-1} \sum_{1 \leq i<j \leq n} \operatorname{Cov}\left(Z_{i}(x), Z_{j}(x)\right) \\
& =\operatorname{Var}\left(Z_{1}(x)\right)+2 n^{-1} \sum_{1 \leq i<j \leq n} \operatorname{Cov}\left(Z_{i}(x), Z_{j}(x)\right) .
\end{aligned}
$$

Let $\Gamma_{n}=n^{-1} \sum_{1 \leq i<j \leq n} \operatorname{Cov}\left(Z_{i}(x), Z_{j}(x)\right)$. By Lemma 4.1 in section 4 ,

$$
\begin{gathered}
\left|\Gamma_{n}\right| \leq C n^{-1} \sum_{1 \leq i<j \leq n}\left|\operatorname{Cov}\left(X_{i}, X_{j}\right)\right| \\
=C n^{-1} \sum_{j=2}^{n} \sum_{i=1}^{j-1}\left|\operatorname{Cov}\left(X_{i}, X_{j}\right)\right| \\
\leq C n^{-1} \sum_{j=2}^{n} \sum_{i:|i-j| \geq 1}\left|\operatorname{Cov}\left(X_{i}, X_{j}\right)\right| \\
\leq C n^{-1} \sum_{j=2}^{n} \sup _{j \geq 1} \sum_{i:|i-j| \geq 1}\left|\operatorname{Cov}\left(X_{i}, X_{j}\right)\right| \\
\leq C \sup _{j \geq 1} \sum_{i:|i-j| \geq 1}\left|\operatorname{Cov}\left(X_{i}, X_{j}\right)\right|=C u(1)<\infty .
\end{gathered}
$$

Hence, $\Gamma_{n}$ is monotonous and bounded about $n$. Therefore, $\Gamma_{n}$ has finite limit as $n \rightarrow \infty$, so that $\lim _{n \rightarrow \infty} n \sigma_{n}^{2}(x)=$ $\sigma^{2}(x)<\infty$, and the assumption (A3) implies that

$$
\sigma_{n}^{2}(x)=O\left(n^{-1}\right), \sigma_{n}^{-2}(x)=O(n) .
$$

Theorem 2.1. Assume that $\left\{X_{i}: i \geq 1\right\}$ is a sequence of identically distributed NA random variables with $E X_{i}=0$ and $E\left|X_{i}\right|^{r}<\infty$ for some $r \geq 1$. Then we have

$$
\hat{\theta}_{n}-\theta^{*}=o\left(n^{-\tau}\right), \text { a.s., }
$$

where (1) when $1 \leq r<2, \tau=1-\frac{1}{r}$; (2) when $r \geq 2$, we can take any $\tau \in[0,1 / 2)$.

Theorem 2.2. Suppose that the assumptions (A1)-(A4) are 
satisfied. Then for any given $x \in \mathbb{R}$, we have

$$
\sup _{u}\left|F_{n}^{x}(u)-\Phi(u)\right| \leq C\left(\gamma_{1 n}^{1 / 3}+\gamma_{2 n}^{1 / 3}+\gamma_{2 n}^{\delta / 2}+u^{1 / 3}\left(q_{n}\right)\right)
$$

where $\Phi(u)$ is the distribution function of $N(0,1)$.

Let $\lambda_{1}$ and $\lambda_{2}$ satisfy $0<\lambda_{1}<\lambda_{2}<1$. Take $q_{n}=$ $\left[n^{\lambda_{1}}\right], p_{n}=\left[n^{\lambda_{2}}\right]$ where $[x]$ denotes the integer part of $x$. In this case, the assumption (A4) is satisfied. Therefore, we have the following corollary.

Corollary 2.1. If the assumptions (A1)-(A3) are satisfied, then for any given $x \in \mathbb{R}$, we have

$$
\frac{1}{\sqrt{n}} \sum_{i=1}^{n}\left(h_{\alpha}\left(X_{i}, x\right)-E h_{\alpha}\left(X_{i}, x\right)\right) \stackrel{d}{\rightarrow} N\left(0, \sigma^{2}(x)\right) .
$$

$$
\sigma^{2}(q)=\lim _{n \rightarrow \infty} n \sigma_{n}^{2}(q)=\lim _{n \rightarrow \infty}\left(n \alpha^{2}\right)^{-1} \operatorname{Var}\left(\sum_{i=1}^{n}\left[X_{i}-q\right]^{+}\right) .
$$

Moreover,

$$
\hat{\theta}_{n}=n^{-1} \sum_{i=1}^{n} h_{\alpha}\left(X_{i}, q\right)+o\left(n^{-\tau}\right), \text { a.s. }
$$

for any $\tau \in(0,1 / 2)$.

(ii) If $q>q^{-}$, then the limit distribution of $n^{1 / 2}\left(\hat{\theta}_{n}-\theta^{*}\right)$ is not a normal distribution.

Remark 2.1. Recall that $X$ is a loss variable of financial asset or portfolio. In practice, the loss variable is usually continuous, so that $q=q^{-}$in Theorem 2.3.

Remark 2.2. Let $\tilde{\theta}_{n}=n^{-1} \sum_{i=1}^{n} h_{\alpha}\left(X_{i}, q\right)$. Note that $q=\operatorname{VaR}_{\alpha}(X)$. Then

$$
\begin{aligned}
\tilde{\theta}_{n} & =n^{-1} \sum_{i=1}^{n}\left(q+\alpha^{-1}\left[X_{i}-q\right]^{+}\right) \\
& =\operatorname{VaR}_{\alpha}(X)+\frac{1}{n \alpha} \sum_{i=1}^{n}\left[X_{i}-\operatorname{VaR}_{\alpha}(X)\right]^{+},
\end{aligned}
$$

it is a common estimator of CVaR. Therefore, the equations (17), (18) and (21) establish the asymptotic relations of the
Theorem 2.3. Let $q=\operatorname{VaR}_{\alpha}(X), q^{-}=\sup \{x: F(x) \leq 1-$ $\alpha$ \}. If (A1)-(A3) are satisfied, we have

$$
\hat{\theta}_{n}=\inf _{x \in\left[q^{-}, q\right]} n^{-1} \sum_{i=1}^{n} h_{\alpha}\left(X_{i}, x\right)+o_{p}\left(n^{-1 / 2}\right) .
$$

There are two cases to consider:

(i) If $q=q^{-}$, we have

$$
\hat{\theta}_{n}=n^{-1} \sum_{i=1}^{n} h_{\alpha}\left(X_{i}, q\right)+o_{p}\left(n^{-1 / 2}\right)
$$

and

$$
n^{1 / 2}\left(\hat{\theta}_{n}-\theta^{*}\right) \stackrel{d}{\rightarrow} N\left(0, \sigma^{2}(q)\right)
$$

where

optimized type CVaR estimator $\tilde{\theta}_{n}$ and the common CVaR estimator $\tilde{\theta}_{n}$.

Remark 2.3. To give the confidence interval of CVaR $\theta^{*}$ by (19), we need to estimate the variance $\sigma^{2}(q)$. Let $\hat{q}_{n}=\widehat{F}_{n}^{-1}(1-\alpha)$ where $\hat{F}_{n}^{-1}(x)$ is empirical distribution function of sample $X_{1}, X_{2}, \cdots, X_{n}$. Denote $V_{i}=\left[X_{i}-\right.$ $\left.\hat{q}_{n}\right]^{+}, i=1,2, \cdots, n$. From (20), it is easy to get the estimation of $\sigma^{2}(q)$ as following

$$
\hat{\sigma}^{2}(q)=\frac{1}{(n \alpha)^{2}} \sum_{i=1}^{n}\left(V_{i}-\bar{V}\right)^{2},
$$

where $\bar{V}=\frac{1}{n} \sum_{i=1}^{n} V_{i}$

\section{Proof of Theorem 2.1}

From the definition of $\hat{\theta}_{n}$ and $\theta^{*}$, we have

$$
\begin{gathered}
n^{\tau}\left|\hat{\theta}_{n}-\theta^{*}\right| \\
=n^{\tau}\left|\left(\hat{\theta}_{n}-\theta^{*}\right) I_{\left(\widehat{\theta}_{n}-\theta^{*} \geq 0\right)}+\left(\hat{\theta}_{n}-\theta^{*}\right) I_{\left(\widehat{\theta}_{n}-\theta^{*}<0\right)}\right| \\
\leq n^{\tau}\left|\hat{\theta}_{n}-\theta^{*}\right| I_{\left(\widehat{\theta}_{n}-\theta^{*} \geq 0\right)}+n^{\tau}\left|\hat{\theta}_{n}-\theta^{*}\right| I_{\left(\widehat{\theta}_{n}-\theta^{*}<0\right)} \\
=n^{\tau}\left|\inf _{x \in \mathbb{R}} \frac{1}{n} \sum_{\mathrm{i}=1}^{\mathrm{n}} h_{\alpha}\left(X_{i}, x\right)-\inf _{y \in \mathbb{R}} E h_{\alpha}\left(X_{1}, y\right)\right| I_{\left(\widehat{\theta}_{n}-\theta^{*} \geq 0\right)} \\
+n^{\tau}\left|\inf _{x \in \mathbb{R}} \frac{1}{n} \sum_{\mathrm{i}=1}^{\mathrm{n}} h_{\alpha}\left(X_{i}, x\right)-\inf _{y \in \mathbb{R}} E h_{\alpha}\left(X_{1}, y\right)\right| I_{\left(\widehat{\theta}_{n}-\theta^{*}<0\right)} \\
=n^{\tau} \inf _{x \in \mathbb{R}}\left|\frac{1}{n} \sum_{\mathrm{i}=1}^{\mathrm{n}} h_{\alpha}\left(X_{i}, x\right)-\inf _{y \in \mathbb{R}} E h_{\alpha}\left(X_{1}, y\right)\right| I_{\left(\widehat{\theta}_{n}-\theta^{*} \geq 0\right)} \\
+n^{\tau} \inf _{y \in \mathbb{R}}\left|E h_{\alpha}\left(X_{1}, y\right)-\inf _{x \in \mathbb{R}} \frac{1}{n} \sum_{\mathrm{i}=1}^{\mathrm{n}} h_{\alpha}\left(X_{i}, x\right)\right| I_{\left(\widehat{\theta}_{n}-\theta^{*}<0\right)} \\
\leq n^{\tau} \inf _{x \in \mathbb{R}}\left|\frac{1}{n} \sum_{\mathrm{i}=1}^{\mathrm{n}} h_{\alpha}\left(X_{i}, x\right)-E h_{\alpha}\left(X_{1}, x\right)+E h_{\alpha}\left(X_{1}, x\right)-\inf _{y \in \mathbb{R}} E h_{\alpha}\left(X_{1}, y\right)\right| \\
+n^{\tau} \inf _{y \in \mathbb{R}}\left|E h_{\alpha}\left(X_{1}, y\right)-\frac{1}{n} \sum_{\mathrm{i}=1}^{\mathrm{n}} h_{\alpha}\left(X_{i}, y\right)+\frac{1}{n} \sum_{\mathrm{i}=1}^{\mathrm{n}} h_{\alpha}\left(X_{i}, y\right)-\inf _{x \in \mathbb{R}} \frac{1}{n} \sum_{\mathrm{i}=1}^{\mathrm{n}} h_{\alpha}\left(X_{i}, x\right)\right| \\
\leq \inf _{x \in \mathbb{R}}\left\{n^{\tau}\left|\frac{1}{n} \sum_{\mathrm{i}=1}^{\mathrm{n}} h_{\alpha}\left(X_{i}, x\right)-E h_{\alpha}\left(X_{1}, x\right)\right|+n^{\tau}\left|E h_{\alpha}\left(X_{1}, x\right)-\inf _{y \in \mathbb{R}} E h_{\alpha}\left(X_{1}, y\right)\right|\right\}
\end{gathered}
$$




$$
+\inf _{y \in \mathbb{R}}\left\{n^{\tau}\left|E h_{\alpha}\left(X_{1}, y\right)-\frac{1}{n} \sum_{\mathrm{i}=1}^{\mathrm{n}} h_{\alpha}\left(X_{i}, y\right)\right|+n^{\tau}\left|\frac{1}{n} \sum_{\mathrm{i}=1}^{\mathrm{n}} h_{\alpha}\left(X_{i}, y\right)-\inf _{x \in \mathbb{R}} \frac{1}{n} \sum_{\mathrm{i}=1}^{\mathrm{n}} h_{\alpha}\left(X_{i}, x\right)\right|\right\}
$$

From Theorem 1 and related contents of Rockafellar and Uryasev [15], we know that $E h_{\alpha}\left(X_{1}, y\right)$ and $\frac{1}{n} \sum_{i=1}^{n} h_{\alpha}\left(X_{i}, y\right)$ are convex function about $y$. Hence, there exist $y_{1} \in R$ and $y_{2} \in R$ such that

$$
E h_{\alpha}\left(X_{1}, y\right)=\inf _{y \in \mathbb{R}} E h_{\alpha}\left(X_{1}, y_{1}\right), \frac{1}{n} \sum_{\mathrm{i}=1}^{\mathrm{n}} h_{\alpha}\left(X_{i}, y_{2}\right)=\inf _{x \in \mathbb{R}} \frac{1}{n} \sum_{\mathrm{i}=1}^{\mathrm{n}} h_{\alpha}\left(X_{i}, x\right) .
$$

Combining (24) and (25), we have

$$
\begin{gathered}
n^{\tau}\left|\hat{\theta}_{n}-\theta^{*}\right| \\
\leq n^{\tau}\left|\frac{1}{n} \sum_{\mathrm{i}=1}^{\mathrm{n}} h_{\alpha}\left(X_{i}, y_{1}\right)-E h_{\alpha}\left(X_{1}, y_{1}\right)\right|+n^{\tau}\left|E h_{\alpha}\left(X_{1}, y_{2}\right)-\frac{1}{n} \sum_{\mathrm{i}=1}^{\mathrm{n}} h_{\alpha}\left(X_{i}, y_{2}\right)\right| \\
\leq n^{\tau-1}\left|\sum_{\mathrm{i}=1}^{\mathrm{n}}\left[h_{\alpha}\left(X_{i}, y_{1}\right)-E h_{\alpha}\left(X_{1}, y_{1}\right)\right]\right|+n^{\tau-1}\left|\sum_{\mathrm{i}=1}^{\mathrm{n}}\left[h_{\alpha}\left(X_{i}, y_{2}\right)-E h_{\alpha}\left(X_{1}, y_{2}\right)\right]\right|
\end{gathered}
$$

Therefore, to prove the Theorem we only need to prove that, for any given $x \in \mathbb{R}$,

$$
\frac{1}{n^{1-\tau}} \sum_{i=1}^{n}\left[h_{\alpha}\left(X_{i}, x\right)-E h_{\alpha}\left(X_{i}, x\right)\right] \stackrel{\text { a.s. }}{\rightarrow} 0 .
$$

(1) For $1 \leq r<2$, by Theorem 2 in Su and Wang [21], we know (27) is true, and $\tau=1-1 / r$.

(2) For $r \geq 2$, take $p \in[1,2)$ and let $\tau=1-1 / p$. Then $E\left|X_{1}\right|^{p} \leq\left(E\left|X_{1}\right|^{r}\right)^{p / r}<\infty$ and $0 \leq \tau<1 / 2$. Hence

$$
\left|\operatorname{Cov}\left(h_{\alpha}\left(X_{1}, x\right), h_{\alpha}\left(X_{2}, x\right)\right)\right| \leq \alpha^{-2}\left|\operatorname{Cov}\left(X_{1}, X_{2}\right)\right| .
$$

Proof Recall that $h_{\alpha}\left(X_{i}, x\right)=x+\alpha^{-1}\left[X_{i}-x\right]^{+}$, have

$$
\operatorname{Cov}\left(h_{\alpha}\left(X_{1}, x\right), h_{\alpha}\left(X_{2}, x\right)\right)=\alpha^{-2} \operatorname{Cov}\left(\left[X_{1}-x\right]^{+},\left[X_{2}-x\right]^{+}\right) .
$$

From Hoeffding Lemma in Lehmamn [9],

$$
\begin{aligned}
& \operatorname{Cov}\left(\left[X_{1}-x\right]^{+},\left[X_{2}-x\right]^{+}\right) \\
& =\int_{-\infty}^{\infty} \int_{-\infty}^{\infty}\left\{P\left(\left[X_{1}-x\right]^{+} \leq u,\left[X_{2}-x\right]^{+} \leq v\right)-P\left(\left[X_{1}-x\right]^{+} \leq u\right) P\left(\left[X_{2}-x\right]^{+} \leq v\right)\right\} d u d v \\
& =\int_{0}^{\infty} \int_{0}^{\infty}\left\{P\left(\left[X_{1}-x\right]^{+} \leq u,\left[X_{2}-x\right]^{+} \leq v\right)-P\left(\left[X_{1}-x\right]^{+} \leq u\right) P\left(\left[X_{2}-x\right]^{+} \leq v\right)\right\} d u d v .
\end{aligned}
$$

When $u \geq 0$ and $v \geq 0$, it is easy to know the following event equations

$$
\begin{aligned}
& \left\{\left[X_{1}-x\right]^{+} \leq u\right\}=\left\{X_{1}-x \leq u\right\},\left\{\left[X_{2}-x\right]^{+} \leq v\right\}=\left\{X_{2}-x \leq v\right\}, \\
& \left\{\left[X_{1}-x\right]^{+} \leq u,\left[X_{2}-x\right]^{+} \leq v\right\}=\left\{X_{1}-x \leq u, X_{2}-x \leq v\right\} .
\end{aligned}
$$

Hence

$$
\begin{aligned}
& \operatorname{Cov}\left(\left[X_{1}-x\right]^{+},\left[X_{2}-x\right]^{+}\right) \\
& =\int_{0}^{\infty} \int_{0}^{\infty}\left\{P\left(X_{1}-x \leq u, X_{2}-x \leq v\right)-P\left(X_{1}-x \leq u\right) P\left(X_{2}-x \leq v\right)\right\} d u d v \\
& =\int_{0}^{\infty} \int_{0}^{\infty}\left\{P\left(X_{1} \leq u+x, X_{2} \leq v+x\right)-P\left(X_{1} \leq u+x\right) P\left(X_{2} \leq v+x\right)\right\} d u d v \\
& =\int_{x}^{\infty} \int_{x}^{\infty}\left\{P\left(X_{1} \leq u, X_{2} \leq v\right)-P\left(X_{1} \leq u\right) P\left(X_{2} \leq v\right)\right\} d u d v
\end{aligned}
$$

Due to NA, $P\left(X_{1} \leq u, X_{2} \leq v\right)-P\left(X_{1} \leq u\right) P\left(X_{2} \leq v\right) \leq 0$ for any $u, v \in \mathbb{R}$, so we get

$$
\begin{aligned}
& \left|\operatorname{Cov}\left(\left[X_{1}-x\right]^{+},\left[X_{2}-x\right]^{+}\right)\right| \\
& =\left|\int_{x}^{\infty} \int_{x}^{\infty}\left\{P\left(X_{1} \leq u, X_{2} \leq v\right)-P\left(X_{1} \leq u\right) P\left(X_{2} \leq v\right)\right\} d u d v\right| \\
& \leq\left|\int_{-\infty}^{\infty} \int_{-\infty}^{\infty}\left\{P\left(X_{1} \leq u, X_{2} \leq v\right)-P\left(X_{1} \leq u\right) P\left(X_{2} \leq v\right)\right\} d u d v\right| \\
& =\left|\operatorname{Cov}\left(X_{1}, X_{2}\right)\right| .
\end{aligned}
$$

Combine (29) with (32) yields (28). Complete the proof. Lemma 4.2. (Luo, [10]) Assume that $E|X|^{r}<\infty$ for some $r>0$. Then there exists a positive constant $\mathrm{C}$ no depending on $x$ such that

$$
E\left|h_{\alpha}(X, x)-E h_{\alpha}(X, x)\right|^{r} \leq C E|X|^{r} .
$$


Lemma 4.3. (Yang, [26]) Suppose that $\left\{X_{i}: i \geq 1\right\}$ is a NA random sequence which satisfies $E X_{i}=0$ and $E\left|X_{i}\right|^{r}<\infty$ for some $r>1$. Then there exists a positive constant $\mathrm{C}$ no depending on $n$ such that

(1) for $1<r \leq 2$,

$$
E \max _{1 \leq k \leq n}\left|\sum_{i=1}^{k} X_{i}\right|^{r} \leq C \sum_{i=1}^{n} E\left|X_{i}\right|^{r}
$$

(2) for $r>2$,

$$
E \max _{1 \leq k \leq n}\left|\sum_{i=1}^{k} X_{i}\right|^{r} \leq C\left(\sum_{i=1}^{n} E\left|X_{i}\right|^{r}+\left(\sum_{i=1}^{n} E X_{i}^{2}\right)^{\mathrm{r} / 2}\right)
$$

Lemma 4.4. (Yang, [27]) Let $\left\{X_{j}: j \geq 1\right\}$ be a NA random sequence, $\left\{a_{j}: j \geq 1\right\}$ be a real-value constant sequence, $1=m_{0}<m_{1}<m_{2}<\cdots<m_{\tau}=n \quad$. Denote $\quad Y_{l}=$
$\sum_{j=m_{l-1}+1}^{m_{l}} a_{j} X_{j}$ for $1 \leq l \leq k$. Then

$$
\begin{gathered}
\left|E \exp \left(i t \sum_{l=1}^{k} Y_{l}\right)-\prod_{l=1}^{k} E \exp \left(i t Y_{l}\right)\right| \leq \\
4 t^{2} \sum_{1 \leq s<j \leq n}\left|a_{s} a_{j}\right|\left|\operatorname{Cov}\left(X_{s}, X_{j}\right)\right| .
\end{gathered}
$$

For any given $x \in \mathbb{R}$, let $Y_{i}=\sigma_{n}^{-1}(x) n^{-1}\left(Z_{i}(x)-\right.$ $\left.E Z_{i}(x)\right), i=1,2, \cdots, n$, so that $S_{n}(x)=\sum_{i=1}^{n} Y_{i}$. Let $k_{n}=$ $\left[n /\left(p_{n}+q_{n}\right)\right]$, where $[x]$ denotes the integer part of $x$ as mentioned before. Then $S_{n}$ may be split as $S_{n}=S_{n}^{\prime}+S_{n}^{\prime \prime}+$ $S_{n}^{\prime \prime \prime}$, where

$$
\begin{gathered}
S_{n}^{\prime}=\sum_{m=1}^{k_{n}} y_{n, m}, S_{n}^{\prime \prime}=\sum_{m=1}^{k_{n}} y_{n, m}^{\prime}, S_{n}^{\prime \prime \prime}=y_{n, k_{n}+1}^{\prime}, \\
y_{n, m}=\sum_{i=t_{m}}^{t_{m}+p_{n}-1} Y_{i}, y_{n, m}^{\prime}=\sum_{i=l_{m}}^{l_{m}+q_{n}-1} Y_{i}, y_{n, k_{n}+1}^{\prime}=\sum_{i=k_{n}\left(p_{n}+q_{n}\right)+1}^{n} Y_{i}, \\
t_{m}=(m-1)\left(p_{n}+q_{n}\right)+1, l_{m}=(m-1)\left(p_{n}+q_{n}\right)+p_{n}+1, m=1,2, \cdots, k_{n} .
\end{gathered}
$$

Lemma 4.5. Assume that the assumptions (A1)-(A4) are satisfied. Then

$$
\begin{gathered}
E\left(S_{n}^{\prime \prime}\right)^{2} \leq C \gamma_{1 n}, E\left(S_{n}^{\prime \prime \prime}\right)^{2} \leq C \gamma_{2 n} \\
P\left(\left|S_{n}^{\prime \prime}\right| \geq \gamma_{1 n}^{1 / 3}\right) \leq C \gamma_{1 n}^{1 / 3}, P\left(\left|S_{n}^{\prime \prime \prime}\right| \geq \gamma_{2 n}^{1 / 3}\right) \leq C \gamma_{2 n}^{1 / 3} .
\end{gathered}
$$

Proof From (13), it is easy to get that

$$
\begin{aligned}
E\left(Y_{i}^{2}\right) & =E\left\{\sigma_{n}^{-1}(x) n^{-1}\left(Z_{i}(x)-E Z_{i}(x)\right)\right\}^{2} \\
& =\sigma_{n}^{-2} n^{-2} E\left(Z_{i}(x)-E Z_{i}(x)\right)^{2} \\
& \leq C \sigma_{n}^{-2}(x) n^{-2} \\
& \leq C n^{-1} .
\end{aligned}
$$

By Lemma 4.3, we can get that

$$
E\left(S_{n}^{\prime \prime}\right)^{2}=E\left(\sum_{m=1}^{k_{n}} y_{n, m}^{\prime}\right)^{2} \leq C \sum_{m=1}^{k_{n}} E\left|y_{n, m}^{\prime}\right|^{2} \leq C \sum_{m=1}^{k_{n}} \sum_{i=l_{m}}^{l_{m}+q_{n}-1} E\left|Y_{i}\right|^{2} .
$$

Using (37), (38) and (9) yields that

$E\left(S_{n}^{\prime \prime}\right)^{2} \leq C k_{n} q_{n} n^{-1} \leq \frac{C q_{n}}{p_{n}+q_{n}} \leq \frac{C q_{n} p_{n}^{-1}}{1+q_{n} p_{n}^{-1}} \leq C q_{n} p_{n}^{-1}=C \gamma_{1 n}$.

Note that $n-k_{n}\left(p_{n}+q_{n}\right)<p_{n}+q_{n}$, we have

$$
\begin{aligned}
E\left(S_{n}^{\prime \prime \prime}\right)^{2} & \leq C \sum_{i=k_{n}\left(p_{n}+q_{n}\right)+1}^{n} E\left(Y_{i}^{2}\right) \\
& \leq C\left(n-k_{n}\left(p_{n}+q_{n}\right)\right) n^{-1} \\
& \leq C\left(p_{n}+q_{n}\right) n^{-1} \\
& \leq C\left(1+q_{n} p_{n}^{-1}\right) p_{n} n^{-1} \\
& \leq C \gamma_{2 n} .
\end{aligned}
$$

immediately yielded form (35). Complete the proof.

Let $s_{n}^{2}=\sum_{m=1}^{k_{n}} \operatorname{Var}\left(y_{n, m}\right)$. We have the following lemma. Lemma 4.6. Under (A1)-(A4), have

$$
\left|s_{n}^{2}-1\right| \leq C\left\{\gamma_{1 n}^{1 / 2}+\gamma_{2 n}^{1 / 2}+u\left(q_{n}\right)\right\}
$$

Proof Because that $E\left(S_{n}^{\prime}\right)^{2}=\sum_{i=1}^{k_{n}} \operatorname{Var}\left(y_{n, m}\right)+$ $2 \sum_{1 \leq i<j \leq k_{n}} \operatorname{Cov}\left(y_{n, i}, y_{n, j}\right)$, we have that

$$
s_{n}^{2}-1=\left(E\left(S_{n}^{\prime}\right)^{2}-1\right)-2 \sum_{1 \leq i<j \leq k_{n}} \operatorname{Cov}\left(y_{n, i}, y_{n, j}\right) \text {. }
$$

Note that $E\left(S_{n}\right)=0$ and $E\left(S_{n}\right)^{2}=\operatorname{Var}\left(S_{n}\right)=1$, we know that

Therefore, (35) holds. By the Markov inequality, (36) is

$$
E\left(S_{n}^{\prime}\right)^{2}=E\left[S_{n}-\left(S_{n}^{\prime \prime}+S_{n}^{\prime \prime \prime}\right)\right]^{2}=1+E\left(S_{n}^{\prime \prime}+S_{n}^{\prime \prime \prime}\right)^{2}-2 E\left[S_{n}\left(S_{n}^{\prime \prime}+S_{n}^{\prime \prime \prime}\right)\right],
$$

hence, from Lemma 4.5,

$$
\begin{aligned}
\left|E\left(S_{n}^{\prime}\right)^{2}-1\right| & =\left|E\left(S_{n}^{\prime \prime}+S_{n}^{\prime \prime \prime}\right)^{2}-2 E\left[S_{n}\left(S_{n}^{\prime \prime}+S_{n}^{\prime \prime \prime}\right)\right]\right| \\
& \leq 2\left(E\left|S_{n}^{\prime \prime}\right|^{2}+E\left|S_{n}^{\prime \prime \prime}\right|^{2}\right)+2\left(E\left|S_{n}\right|^{2}\right)^{1 / 2}\left(E\left|S_{n}^{\prime \prime}+S_{n}^{\prime \prime \prime}\right|^{2}\right)^{1 / 2} \\
& \leq 2\left(E\left|S_{n}^{\prime \prime}\right|^{2}+E\left|S_{n}^{\prime \prime \prime}\right|^{2}\right)+2\left(E^{1 / 2}\left|S_{n}^{\prime \prime}\right|^{2}+E^{1 / 2}\left|S_{n}^{\prime \prime \prime}\right|^{2}\right) \\
& \leq C\left(\gamma_{1 n}^{1 / 2}+\gamma_{2 n}^{1 / 2}\right) .
\end{aligned}
$$


And

$$
\begin{aligned}
\left|\sum_{1 \leq i<j \leq k_{n}} \operatorname{Cov}\left(y_{n, i}, y_{n, j}\right)\right| & \leq \sum_{1 \leq i<j \leq k_{n}} \sum_{s=t_{i}}^{t_{i}+p_{n}-1} \sum_{t=t_{j}}^{t_{j}+p_{n}-1}\left|\operatorname{Cov}\left(Y_{s}, Y_{t}\right)\right| \\
& \leq C \sum_{i=1}^{k_{n}-1} \sum_{j=i+1}^{k_{n}} \sum_{s=t_{i}}^{t_{i}+p_{n}-1} \sum_{t=t_{j}}^{t_{j}+p_{n}-1} n^{-1}\left|\operatorname{Cov}\left(X_{s}, X_{t}\right)\right| \\
& \leq C n^{-1} \sum_{i=1}^{k_{n}-1} \sum_{s=t_{i}}^{t_{i}+p_{n}-1} \sup _{j \geq 1} \sum_{t:|t-j| \geq q_{n}}\left|\operatorname{Cov}\left(X_{j}, X_{t}\right)\right| \\
& \leq C u\left(q_{n}\right) .
\end{aligned}
$$

Therefore, (39) holds from (40)-(42). Complete the proof.

Lemma 4.7. (Yang, [27]) Suppose that $\left\{\zeta_{n}: n \leq 1\right\}$ and $\left\{\eta_{n}: n \leq 1\right\}$ are two random sequences, $\left\{\gamma_{n}: n \leq 1\right\}$ is a positive constant sequence, and $\gamma_{n} \rightarrow 0$. If

$$
\sup _{u}\left|F_{\zeta_{n}}(u)-\Phi(u)\right| \leq C \gamma_{n}
$$

then for any $\epsilon>0$, have

$$
\begin{aligned}
\sup _{u}\left|F_{\zeta_{n}+\eta_{n}}(u)-\Phi(u)\right| & \leq C\left(\gamma_{n}+\epsilon+P\left(\left|\eta_{n}\right| \geq \epsilon\right)\right) . \\
& \sup _{u}\left|\hat{F}_{n}^{x}(u)-\Phi(u)\right| \\
& \leq \sup _{u}\left|\hat{F}_{n}^{x}(u)-\hat{G}_{n}^{x}(u)\right|+\sup _{u}\left|\hat{G}_{n}^{x}(u)-\Phi\left(u / s_{n}\right)\right|+\sup _{u}\left|\Phi\left(u / s_{n}\right)-\Phi(u)\right| \\
& =\sup _{u}\left|\hat{F}_{n}^{x}(u)-\hat{G}_{n}^{x}(u)\right|+\sup _{u}\left|\hat{G}_{n}^{x}\left(u / s_{n}\right)-\Phi\left(u / s_{n}\right)\right|+\sup _{u}\left|\Phi\left(u / s_{n}\right)-\Phi(u)\right| \\
& =J_{1 n}+J_{2 n}+J_{3 n} .
\end{aligned}
$$

First to estimate $J_{2 n}$. By Lemma 4.3 we know

$$
\begin{aligned}
\sum_{m=1}^{k_{n}} E\left|\eta_{n, m}\right|^{2+\delta} & \leq C \sum_{m=1}^{k_{n}}\left(\sum_{i=t_{m}}^{t_{m}+p_{n}-1} E\left|Y_{i}\right|^{2+\delta}+\left(\sum_{i=t_{m}}^{t_{m}+p_{n}-1} E Y_{i}^{2}\right)^{(2+\delta) / 2}\right) \\
& \leq C \sum_{m=1}^{k_{n}}\left(\sum_{i=t_{m}}^{t_{m}+p_{n}-1} \sigma_{n}^{-(2+\delta)} n^{-(2+\delta)}+\left(\sum_{i=t_{m}}^{t_{m}+p_{n}-1} n^{-1}\right)^{(2+\delta) / 2}\right) \\
& \leq C\left(\sum_{i=1}^{n} n^{-1-\delta / 2}+\left(p_{n} n^{-1}\right)^{(1+\delta / 2)}\right) \\
& \leq C\left(n^{-\delta / 2}+k_{n}\left(p_{n} n^{-1}\right)^{(1+\delta / 2)}\right) \\
& \leq C\left(n^{-\delta / 2}+\left(p_{n} n^{-1}\right)^{\delta / 2}\right) \\
& \leq C\left(p_{n} n^{-1}\right)^{\delta / 2} \\
& =C \gamma_{2 n}^{\delta / 2} .
\end{aligned}
$$

Note that $B_{n}^{2}=s_{n}^{2} \rightarrow 1$ by Lemma 4.6. From the BerryEsseen theorem, we get that

$$
\begin{aligned}
J_{2 n} \leq C B_{n}^{-(2+\delta)} \sum_{m}^{k_{n}} E\left|y_{n, m}\right|^{2+\delta} & \leq C \gamma_{2 n}^{\delta / 2} \cdot(45) \\
J_{1 n} & \leq \int_{-T}^{T}\left|\frac{\varphi(t)-\psi(t)}{t}\right| d y+T \sup _{u} \int_{|y| \leq c / T}\left|\tilde{G}_{n}^{x}(u+y)-\tilde{G}_{n}^{x}(u)\right| d y .
\end{aligned}
$$

Note that

$$
\psi(t)=E \exp \left(i t T_{n}\right)=E \exp \left(i t \sum_{m=1}^{k_{n}} \eta_{n, m}\right)=\prod_{m=1}^{k_{n}} E \exp \left(i t \eta_{n, m}\right)=\prod_{m=1}^{k_{n}} E \exp \left(i t y_{n, m}\right),
$$

and utilize Lemma 4.4

$$
\begin{aligned}
|\varphi(t)-\psi(t)| & =\left|E \exp \left(i t \sum_{m=1}^{k_{n}} y_{n, m}\right)-\prod_{m=1}^{k_{n}} E \exp \left(i t y_{n, m}\right)\right| \\
& \leq 4 t^{2} \sum_{1 \leq i<j \leq k_{n}} \sum_{u=t_{i}}^{t_{i}+p_{n}-1} \sum_{v=t_{j}}^{t_{j}+p_{n}-1}\left|\operatorname{Cov}\left(Y_{u}, Y_{v}\right)\right| \\
& \leq 4 t^{2} u\left(q_{n}\right) .
\end{aligned}
$$

On the other hand, 


$$
\begin{aligned}
\sup _{u}\left|\tilde{G}_{n}^{x}(u+y)-\tilde{G}_{n}^{x}(u)\right| & =\sup _{u}\left|G_{n}^{x}\left((u+y) / s_{n}\right)-G_{n}^{x}\left(u / s_{n}\right)\right| \\
& \leq \sup _{u}\left|G_{n}^{x}\left((u+y) / s_{n}\right)-\Phi\left((u+y) / s_{n}\right)\right| \\
& +\sup _{u}\left|\Phi\left((u+y) / s_{n}\right)-\Phi\left(u / s_{n}\right)\right|+\sup _{u}\left|G_{n}^{x}\left(u / s_{n}\right)-\Phi\left(u / s_{n}\right)\right| \\
& \leq C\left(\gamma_{2 n}^{\delta / 2}+|y| / s_{n}\right) \leq C\left(\gamma_{2 n}^{\delta / 2}+|y|\right) .
\end{aligned}
$$

Let $T=u^{-1 / 3}\left(q_{n}\right)$ then have

$$
\begin{aligned}
J_{1 n} & \leq C\left(\int_{-T}^{T}|4 t u(q)| d t+T \int_{|y| \leq C / T}\left(\gamma_{2 n}^{\delta / 2}+|y|\right) d y\right) \\
& \leq C\left(u\left(q_{n}\right) T^{2}+\gamma_{2 n}^{\delta / 2}+1 / T\right) \\
& \leq C\left(u^{1 / 3}\left(q_{n}\right)+\gamma_{2 n}^{\delta / 2}\right) .
\end{aligned}
$$

Finally to estimate $J_{3 n}$. By mean value theorem, there exists $\xi_{u} \in\left[u, u / s_{n}\right] \cup\left[u / s_{n}, u\right]$ such that

$$
\begin{aligned}
J_{3 n} & =\sup _{u}\left|\Phi\left(u / s_{n}\right)-\Phi(u)\right| \\
& =\sup _{u} \frac{1}{\sqrt{2 \pi}} e^{-\xi_{u}^{2} / 2}\left|u / s_{n}-u\right| \leq C\left\{\gamma_{1 n}\right. \\
& \leq \frac{\left|s_{n}-1\right|}{\sqrt{2 \pi} s_{n}} \sup _{u}|u| e^{-u^{2} \min \left\{1,1 / s_{n}^{2}\right\} / 2} \\
& =\frac{\left|s_{n}-1\right|}{\sqrt{2 \pi} s_{n}} \frac{1}{\sqrt{\min \left\{1,1 / s_{n}^{2}\right\}}} . \\
& \sup _{u}\left|\tilde{F}_{n}^{x}(u)-\Phi(u)\right| \leq C\left(\gamma_{1 n}^{1 / 2}+\gamma_{2 n}^{1 / 2}+\gamma_{2 n}^{\delta / 2}+u^{1 / 3}\left(q_{n}\right)\right) .
\end{aligned}
$$

By Lemma 4.5 and Lemma 4.7, we have

$$
\begin{aligned}
\sup _{u}\left|F_{n}^{x}(u)-\Phi(u)\right| & \leq C\left(\gamma_{1 n}^{1 / 2}+\gamma_{2 n}^{1 / 2}+\gamma_{1 n}^{1 / 3}+\gamma_{2 n}^{1 / 3}+\gamma_{2 n}^{\delta / 2}+u^{1 / 3}\left(q_{n}\right)\right) \\
& \leq C\left(\gamma_{1 n}^{1 / 3}+\gamma_{2 n}^{1 / 3}+\gamma_{2 n}^{\delta / 2}+u^{1 / 3}\left(q_{n}\right)\right) .
\end{aligned}
$$

Complete the proof.

\section{Proof of Theorem 2.3}

Lemma 5.1. (Shapiro, [19]) Suppose that, for a sequence $\left\{\tau_{n}\right\}$ of positive number converging to infinity, the sequence $\tau_{n}\left\{\hat{f}_{n}(x)-f(x)\right\}$, of random elements of $C(S)$, converges in distribution to a random element $Y(x)$ of $C(S)$. Then

$$
\tau_{n}\left(\inf _{x \in S} \hat{f}_{n}(x)-\inf _{x \in S} f(x)\right) \stackrel{d}{\rightarrow} \inf _{x \in S^{*}(f)} Y(x),
$$

where $S \subseteq \mathbb{R}$ and $f(x): S \rightarrow \mathbb{R}, \hat{f}_{n}(x)$ is sample average of $f(x), S^{*}(f)$ is a set of points that $f(x)$ reaches minimum values in these points.

Lemma 5.2. (Pflug, [14]) Suppose that $F(x)$ is distribution function of random variable $X$. For $\alpha \in(0,1)$, let $a=$ $\sup \{u: F(u) \leq 1-\alpha\}$ and $b=\inf \{u: F(u) \geq 1-\alpha\}$. Then

$$
[a, b]=\operatorname{argmin}\left\{x+\frac{1}{\alpha} E[X-x]^{+}, x \in \mathbb{R}\right\} .
$$

particularly, $\quad F^{-1}(1-\alpha) \in \operatorname{argmin}\left\{x+\frac{1}{\alpha} E[X-x]^{+}: x \in\right.$ $\mathbb{R}\}$.

Proof of Theorem 2.3 From Corollary 2.1, we know that, for any $x \in \mathbb{R}$,

$$
n^{1 / 2}\left[n^{-1} \sum_{i=1}^{n} h_{\alpha}\left(X_{i}, x\right)-E h_{\alpha}(X, x)\right] \stackrel{d}{\rightarrow} N\left(0, \sigma^{2}(x)\right) .
$$

Note that $\left[q^{-}, q\right]=\operatorname{argmin}\left\{E h_{\alpha}(X, x), x \in \mathbb{R}\right\}$ from Lemma 5.2. So, by Lemma 5.1, we have

$$
n^{1 / 2}\left(\hat{\theta}_{\mathrm{n}}-\theta^{*}\right) \stackrel{d}{\rightarrow} \inf _{x \in\left[q^{-}, q\right]} N\left(0, \sigma^{2}(x)\right)
$$

and

$$
\begin{aligned}
\inf _{x \in\left[q^{-}, q\right]}\{ & \left.n^{1 / 2}\left[n^{-1} \sum_{i=1}^{n} h_{\alpha}\left(X_{i}, x\right)-E h_{\alpha}(X, x)\right]\right\} \\
& \stackrel{d}{\rightarrow} \inf _{x \in\left[q^{-}, q\right]} N\left(0, \sigma^{2}(x)\right) .
\end{aligned}
$$

Thus, (56) and (57) imply that

$$
\begin{gathered}
\hat{\theta}_{\mathrm{n}}-\theta^{*}=\inf _{x \in\left[q^{-}, q\right]}\left\{n^{-1} \sum_{i=1}^{n} h_{\alpha}\left(X_{i}, x\right)-E h_{\alpha}(X, x)\right\}+ \\
o_{p}\left(n^{-1 / 2}\right) .
\end{gathered}
$$

Lemma 5.2 tells us that $E h_{\alpha}(X, x)=\inf _{x \in\left[q^{-}, q\right]} E h_{\alpha}(X, x)=\theta^{*}$ for any $x \in\left[q^{-}, q\right]$. Therefore, (58) is equivalent to

$$
\hat{\theta}_{\mathrm{n}}=\inf _{x \in\left[q^{-}, q\right]} n^{-1} \sum_{i=1}^{n} h_{\alpha}\left(X_{i}, x\right)+o_{p}\left(n^{-1 / 2}\right),
$$

that is (17).

When $q=q^{-}$, it is obvious that (18) and (19) hold from (59) and (56), respectively. Let $\eta_{n}=\hat{\theta}_{\mathrm{n}}-$ $n^{-1} \sum_{i=1}^{n} h_{\alpha}\left(X_{i}, q\right)$, then

$$
\eta_{n}=\left(\hat{\theta}_{\mathrm{n}}-\theta^{*}\right)-\left(n^{-1} \sum_{i=1}^{n}\left[h_{\alpha}\left(X_{i}, q\right)-E h_{\alpha}\left(X_{i}, q\right)\right]\right) .
$$

From Theorem 2.1 and (27), we know that $\eta_{n}=$ $o\left(n^{-\tau}\right)$, a.s., so obtain (21). 
When $q>q^{-}$, from (56), the limiting distribution of $n^{1 / 2}\left(\hat{\theta}_{\mathrm{n}}-\theta^{*}\right)$ is a minimum value of a family of normal distributions, so it isn't a normal distribution.

\section{Conclusion}

We know that ARMA models with negative autocorrelation coefficients are NA models, so NA dependent samples are widely existed in practice. Under NA variables, therefore, it is meaningful to discuss the asymptotic properties of the optimized type CVaR estimator proposed by Rockafellar and Uryasev [15] and Trindade et al [23]. We derive the consistency and the asymptotic normality of the estimator, and the consistency rates are $n^{-1 / 2}$ or near to $n^{-1 / 2}$. These results show that the estimatior has good theoretical properties in NA dependent environment. And the confidence interval of CVaR can be given by using the property of asymptotic normality.

\section{Fund}

This research was supported by the National Natural Science Foundation of China [grant number 11461009] and the Natural Science Foundation of Hainan [grant number 117173].

\section{References}

[1] Andrew, E. B., et al. Conditional value-at-risk in portfolio optimization: Coherent but fragile. Operations Research Letters, 2011, 39: 163-171.

[2] Artzner, P., Delbaen, F., Eber, J., Heath, D. Thinking coherently. Risk, 1997, 10: 68-71.

[3] Artzner, P., Delbaen, F., Jean-Marceber. Coherent measures of risk. Mathematical Finance, 1999, 9 (3): 203-228.

[4] Chen, H., et al. Conditional value-at-risk-based optimal spinning reserve for wind integrated power system. Int. Trans. Electr. Energ. Syst., 2016, 26: 1799-1809.

[5] Escanciano, J. C., Mayoral, S. Semiparametric estimation of dynamic conditional expected shortfall models. Int. J. Monetary Economics and Finance, 2008, 1 (2): 106.

[6] Föllmer, H., Schied, A. Stochastic Finance: An Introduction in Discrete Time. Walter De Gruyter Incorporated, 2004.

[7] Gotoh, J., Takano, Y. Newsvendor solutions via conditional value-at-risk minization European. Journal of Operational Reserch, 2007, 170 (1): 80-96.

[8] Legg, S. W., et al. Optimal gas detector placement under uncertainty considering Conditional Value-at-Risk. Journal of Loss Prevention in the Process Industries, 2013, 26: 410-417.

[9] Lehmann, E. L. Some Concepts of Dependence. The Annals of Mathematical Statistics, 1966, 37 (5): 1137-1153.

[10] Luo, Z., Yang, S. The Asymptotic properties of CVaR estimator under $\rho$ mixing sequences. Acta Mathematica Sinica
(Chinese Series), 2013, 56 (6): 851-870.

[11] Luo, Z., Ou, S. The almost sure convergence rate of the estimator of optimized certainty equivalent risk measure under $\alpha$-mixing sequences. Communications in StatisticsTheory and Methods, 2017, 46 (16): 8166-8177.

[12] Mansini, R., Ogryczak, W., Speranza, M. G. Conditional value at risk and related linear programming models for portfolio optimization. Ann Oper Res, 2007, 152: 227-256.

[13] Noyan, N., Rodulf, G. Optimization with Multivariate Conditional Value-at-Risk Constraints. operations research, 2013, 61 (4): 990-1013.

[14] Pflug, G. Some Remarks on the Value-at-Risk and the Conditional Value-at-Risk. Probabilistic Constrained Optimization, 2000: 272-277.

[15] Rochafellar, R. T., Uryasev, S. Optimization of Conditional value-at-risk. The Journal of Risk, 2000, 3 (2): 21-24.

[16] Rockafellar, R. T., Uryasev, S. Conditional value-at-risk for general loss distributions. Research report, 2002.

[17] Roussas, G. G. Asymptotic normality of the kernel estimate of a probability density function under association. Statistics Probability Letters, 2000, 50: 1-12.

[18] Scaillet, O. Nonparametric estimation and sensitivity ananlysis of expected shortfall. Mathematical Finance, 2004, 14 (1): 115-129.

[19] Shapiro, A. Statistical inference of stochastic optimization problem. In: Uryasev. S. (ed), Probabilistic Constrained Optimization: Methodology and Applications, Kluwer Academic Publisher, Dordrecht, 2000: 282-304.

[20] Su, C., Zhao, L. Moment inequalities and weak convergence for negatively associated sequences. Science in China (Series A), 1997, 40 (2): 172-182.

[21] Su, C., Wang, Y. Strong Convergence for IDNA Sequences. Chiness Journal of Applied Probability and Statistics, 1998, 14 (2): 131-140.

[22] Sun, H., et al. Optimal multivariate quota-share reinsurance: A nonparametric mean-CVaR framework. Insurance: Mathematics and Economics, 2017, 72: 197-214.

[23] Trindade, A. A., et al. Financial prediction with constrained tail risk. Journal of Banking and Finance, 2007, 31 (11): 3524-3538.

[24] Wang, C. S, Zhao, Z. Conditional Value-at-Risk: Semiparametric estimation and inference. Journal of Econometrics, 2016, 195: 86-103.

[25] Xing, G, D. et al. Strong Consistency of Conditional Value-atrisk Estimate for $\phi$-mixing Samples. Communications in Statistics-Theory and Methods, 2014, 43: 5105-5113.

[26] Yang, S. Moment inequalities for partial sums of random variables. Science in China (Series A), 2001, 44: 1-6.

[27] Yang, S. Uniformly asymptotic normality of the regression weighted estimator for negatively associated samples. Statistics-Probability letters, 2003: 101-110.

[28] Zhou, X., et al. Moment consistency of estimators in partially linear models under NA samples. Metrika, 2010, 72: 415-432. 